\title{
RADIANT HEAT AN EXCEPTION TO THE SECOND LAW OF THERMO-DYNAMICS.*
}

\author{
By H. T. Eddy, Рh. D.
}

Since the radiation of heat takes place by propagation through space. at a certain finite velocity, and not instantaneously, it is quite possible for occurrences to intervene during the exchange of radiations between two bodies, such as to essentially change the distribution of heat which would otherwise have ultimately taken place.

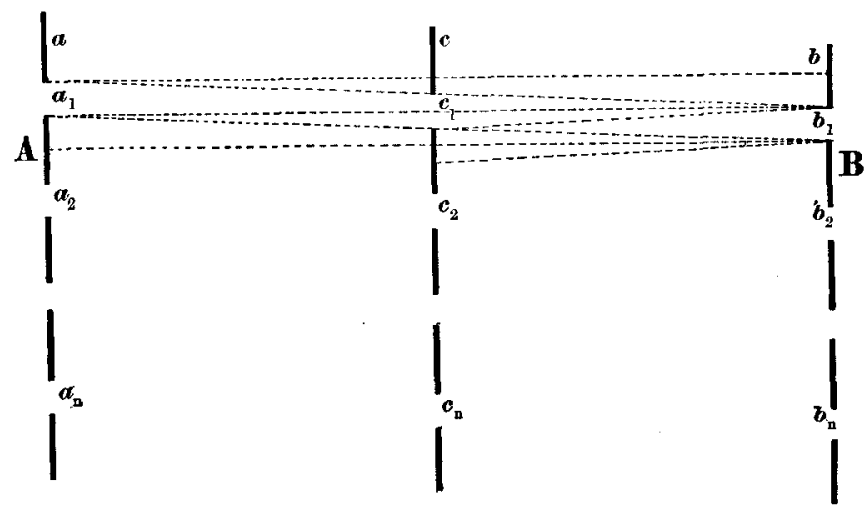

To make this evident, let us first employ a mechanical analogy. In the accompanying figure, let there be three parallel screens $a, b$, and $c$, the latter between the two former, and all three perpendicular to the plane of the paper. Let them be pierced respectively by a series of equidistant apertures $a_{1} a_{2} a_{n}, b_{1} b_{2} b_{n}, c_{1} c_{2} c_{n}$, situated in the plane of the paper, and let these apertures be so placed that $a_{1}, b_{1}, c_{1}$, are upon one straight line, not quite at right angles to the screens; then are $a_{2}$, $b_{2}, c_{2}$, etc., and $a_{\mathrm{n}}, b_{\mathrm{n}}, c_{\mathrm{n}}$, upon lines parallel to $a_{1}, b_{1}, c_{1}$. Now conceive the screens $a, b$, $c$, to have a common uniform velocity, $u$, in the direction from $c_{2}$ to $c_{1}$. Also, let a series of projectiles be discharged from any fixed position, $A$, at the left of the screen $a$, at such instants as to pass the first one through the aperture $a_{1}$, the second through $a_{2}$, etc.,

* From the Proceedings of the Ohio Mechanics' Institute, May 25, 1882. 
and let the direction of discharge be perpendicular to the screens, and the velocity, $v$, such that each one shall just reach the screen $b$ in time to pass through the first aperture of that screen which crosses its patl. Then would the screens $a b c$ in no way interfere with the passage of these projectiles. Let us denote the space at the left of $a$ as the space $A$, and that at the right of $b$ as the space $B$. Then, if there be a continuous discharge of projectiles from all points of the space $A$, only a part of them can pass through the apertures of $a$. Such, however, as succeed in passing $a$, will pass $b$ and $c$ also. Again, let a second discharge of projectiles take place from the space $B$, but directed toward the left perpendicularly to the screens, so that these projectiles move in a precisely opposite direction from those first mentioned. Let the projectiles from $B$ have the common velocity $v^{\prime}$. Such of these projectiles as succeed in passing through the apertures of $b$ will impinge on $c$ at points between its apertures, in case $c$ be placed at a proper distance from $b$. Let the surface of $c$ which faces $b$ be perfectly reflecting, and let the parts between its apertures be either concave, or a series of inclined planes, so directed that each of the projectiles on rebounding will pass back through one of the apertures in $b$. When the velocity $v^{\prime}$ of the projectiles is large compared with that of the screens $u$, the projectiles can be made to return through $b$ very nearly perpendicularly, either by returning each projectile through that aperture from which it started or through some following one.

The paths of the projectiles relative to the screens can be readily found by impressing upon the projectiles, in addition to their velocities $v$ or $v^{\prime}$, a velocity-u, numerically equal and opposed to that of the screens, while the screens themselves are at rest. The composition of these velocities will give the required relative velocity.

In order to apply the mechanical analogy just considered to the case in hand, let us replace the supposed projectiles by radiations which emanate from warm bodies situated in the spaces $A$ and $B$, and let the only radiations at first considered be those in a direction perpendicular to the screen.

It is then evident that with such a series of apertures as are represented in the figure, the screens $a b c$ could be given such a velocity $u$, as, accompanied by reflections from $c$, would transfer radiations from the body $A$ to $B$, unaccompanied by a compensating transfer from $B$ to $A$; and thus the body $B$ would be heated at the expense of $A$. 
Even if radiations at the apertures in $a$ and $b$ be not confined to rays perpendicular to the screens, but take place instead in the manner usual at plane surfaces, it is still evident that the usual interchange of radiations has been effectively interfered with, and that the body $B$ would be heated at the expense of $A$. In case the radiations from the body $B$ are reflected back through the same apertures from which they started, it is quite unnecessary to have the series of apertures in the screen $a$ at equal distances; it is only necessary that the series of apertures in $b$ and $c$ correspond to those in $a$. Indeed, each aperture in $b$ can be conceived to be completely surrounded by a concave semicylindrical reflector attached to $c$, of such a form as to return to $b$ all radiations from it when moving with the velocity $u$. This can certainly by effected if the apertures in $b$ are mere points, and can be closely approximated to when they are small. Now, if there be in this cylinder a proper aperture for the admission of the normal radiations from $A$ through $a$, it is evident that the radiations passing through this aperture from $B$, being oblique, are, when the bodies are of equal temperature, less than those of $A$ passing through the same aperture, according to the well known law of radiations that the intensity is proportional to the cosine of the angle between the ray and the normal to the radiating surface. It is seen that with a sufficiently large value of $u$, it would be possible to overcome any difference of temperature, however great.

In order to form an estimate of the amount by which the radiation from $A$ to $B$ exceeds that escaping from $B$ through $c$, let us suppose that the temperatures of $A$ and $B$ are equal, and that the velocity $v$ of the radiations from $A$ and $B$ is the same; and, further, let the screen $c$ be midway between $a$ and $b$ at a distance $p$ from each. Let the problem be to compute the ratio between the radiations which pass through a given aperture, as $c_{1}$, from $a_{1}$, and from $b_{1}$, respectively, on the supposition that the beat radiates from the equal apertures $a_{1}$ and $b_{1}$, as from plane surfaces, in the usual manner.

Suppose that the linear dimensions of the apertures are infinitesimal compared with $p$, and let the letters $a_{1} b_{1} c_{1}$, considered as numerical magnitudes, designate the areas of the apertures $a_{1} b_{1} c_{1}$ respectively. Let $\theta$ be the angle between a ray and the normal to the surface from which it radiates. Let a sphere of radius $p$ be supposed to be deseribed about some point of $b_{1}$ as a centre, and let $s$ be the area of that part 
of its surface included within the cone of rays passing from the centre to the periphery of the aperture $c_{1}$;

$$
\text { then } \frac{s}{p^{2}}=\frac{c_{1}}{r^{2}} \cos \theta
$$

in which $r$ is the distance passed over by the ray from $b$ to $c$.

$$
\begin{gathered}
\text { Also } p=r \cos \theta \\
\text { therefore } s=c_{1} \cos ^{3} \theta
\end{gathered}
$$

Now the heat radiated from $b_{1}$ is directly proportional to the area $b_{1}$, to the area $s$, and to $\cos \theta$, but inversely proportional to $p^{2}$;

$$
\text { hence } \frac{b_{1} s}{p^{2}} \cos \theta=\frac{b_{1} c_{1}}{p^{2}} \cos ^{4} \theta
$$

is proportional to the heat radiated from $b_{1}$ through $c_{1}$.

Similarly,

$$
\frac{a_{1} c_{1}}{p^{2}}
$$

is proportional to the heat radiated from $a_{1}$ through $c_{1}$, since it passes $c$ normally. Now the heat passing from $b_{1}$ to $c_{1}$ must evidently move in a direction to overtake the aperture $c_{1}$, and to do this it must evidently take a direction such that $\theta$ is defined by the equation

$$
\tan \theta=\frac{2 u}{v,} \text { or } \cos ^{2} \theta=\frac{v^{2}}{v^{2}+4 u^{2}}
$$

Hence, by comparing expressions (4) and (5), and substituting from (6), it appears that the heat radiated from $a_{1}$ through $c_{1}$ is greater than that radiated by an equal surface $b_{1}$ through $c_{1}$, in the ratio of $\left(v^{2}+4 u^{2}\right)^{2}$ to $v^{4}$, in case the temperatures of $a_{1}$ and $b_{1}$ are equal. If the temperature of $a_{1}$ were lower than that of $b_{1}$, this ratio would be diminished; but by increasing $u$, the ratio can still be made to exceed unity, thus confirming the observations previously mare. Neither is it essential that the radiations all take place at the same velocity. The reflectors can be arranged for some one velocity, and they will then send back the radiations to $B$, which have that velocity.

Perhaps the most simple ideal arrangement for effecting the proposed interference $e^{*}$ with the radiations naturally taking place between two bodies, is to suppose the apertures distributed around the circumferences of equal circles, upon three parallel disks fixed upon a common central axis, so that the plane of the paper in the figure becomes the surface of a circular cylinder, in which case the required velocity $u$ can be given to the apertures by simple rotation. Let us for brevity call such an 
arrangement a radiation syren, or simply a syren, as it slightly resembles in its mechanical details the acoustic instrument called by that name. Now, theoretically, no expenditure of energy is necessary to preserve the uniform velocity of the moving parts of this syren; and once started with a sufficiently high velocity of rotation and proper adjustment of reflectors, it would transfer heat from the body $A$ to $B$, regardless of their temperatures, provided no radiations are permitted except those perpendicular to the disks, excluding, of course, all radiations to and from all bodies other than $A$ and $B$. It would also, as before shown, transfer heat from a colder body to a hotter, even though the radiation follow the general law of radiations from plane surfaces.

It is needless to state that the action of the syren, regarded as a possible physical process, is directly at variance with hitherto accepted axioms and conclusions respecting the second law of thermo-dynamics. It is true, we should at first thought be inclined to the belief that the laws of heat should suffer some modification, in case we assume differing rates of propagation not infinite, but we should hardly be prepared to admit the startling conclusions which must flow from such modification, if the physical process just sketched be admitted to be valid; and these I shall now proceed to develop.

I think it may be readily perceived that the axioms of Clausius, upon which he founds the second law, viz., that " heat can not of itself pass from a colder into a hotter body," when applied to radiations, implicitly assumes that the heat is radiated with infinite velocity; for it takes no account of the states of relative rest or motion of the bodies between which heat passes.

The axiom of Thomson, "It is impossible, by means of inanimate material agency, to derive mechanical effect from any portion of matter by cooling it below the temperature of the coldest of surrounding: objects," is obnoxious to the same criticism ; and, as, I have stated elsewhere,* these should not be called axioms at all, since we are not in a position to bring sufficient experience to bear upon them to affirm their validity or want of validity. Indeed, if the process of the syren be adnitted to be possible, we are now in a position to assert that there exists an unexplained contradiction, which does not permit us to consider them as applicable to radiations of heat propagated at finite velocities.

*Thero-dynamics, New York, 1879. 
What, it seems to me, the statements of Clausius and Thonson just quoted, really asserted, was the historical fact that at the date when they were made no one had as yet invented any machine, or discovered any principle on which it was possible to construct a machine, which could successfully accomplish what these said had not been done; and it was further implied that no such machine could probahly ever be invented, nor any such principle discovered.

In complete accord with this statement is that of Kirchhoff, made in his lectures upon the Theory of Heat during the summer semester of 1880 , in which he said, if correctly reported, that the second law can not be (at present) proved; but it, so far, has never been found in disagreement with experience.

It is well known that Maxwell has proposed a process to accomplish this very object; namely, to transfer heat from a colder to a hotter body, in the following manner: If we suppose minute beings, endowed with senses sufficiently acute, and having a corresponding agility to. guard minute openings in the diaphragm separating two portions of the same gas, which openings are only large enough for a single molecule to pass at once, they would be able, without expenditure of energy, to open and close the openings in such a way as to allow each molecule impinging at an opening to pass through or not, as they should choose. If they permitted only those molecules having more than the mean vis viva to pass in one direction, and only those having less than the mean to pass in the opposite direction, then the gas in one side of the diaphragm would gain energy at the expense of that on the other side. That this process is actually at present beyond human ability, does not show that we may not at some future time be able to accomplish what Maxwell proposed. If this be admitted, then the conclusions which I shall draw later from the lack of generality in the second law of thermo-dynamics flow to a limited extent from the possibility of this process. But Maxwell's process assumes the kinetic theory of gases as its basis, and stands or falls with it. And if the second law is a necessary ultimate mechanical prineiple, holding for all bodies great and small, the above consequence of the kinetic theory of gases being in contradiction to the second law, is fatal to the validity of the kinetic theory. But I do not now so regard the second law. I am eompelled to regard it as merely an approximation in the case of radiations, and to regard it in general with Maxwell and with Boltzmann,* as merely

*Wien. Sitrb., Bände LXXVI, LXXVIII. 
the mean result flowing from the laws of propability, though it had previously seemed to me possible to show it to depend upon funda- mental considerations, respecting the nature of heat as a form of energy as was stated in my work previously referred to.

To advert to the consequences which are thus made to flow from the established fact of the finite velocity of radiant heat, we may mention that if the law of the dissipation of energy is no longer to be regarded as of universal validity, it being obviated by the process of the syren, it is just as possible to avail ourselves of the heat stored in cold bodies as in hot ones, and thus to employ the heat of a glacier to drive a steam engine, or to perform other like feats heretofore regarded as impossibilities. When I say it is just as possible, I do not imply that it is now just as practicable, or perhaps, ever will be so. That these observations are just is seen when we reflect that the process of the syren sinply heats a given body at the expense of any other, regardless of temperatures, by a method requiring the expenditure of no energy. It thus appears that it is possible to avail ourselves of the heat existing in bodies beiow the lowest thermometric levels of surrounding objects.

It may be objected that the syren renders a perpetual motion a possibility. That depends upon the definition of perpetual motion which we adopt. In the popular acceptation of that term, the process of the syren, as well as that of Maxwell, would make something near that possible. But when correctly viewed, the process of the syren does not imply the possibility of a perpetual motion, any more than does combustion or using the available energy of any chemical process. It simply proposes to employ the finite amount of energy existing in a given borly, in the form of heat, in a given way.

It is admitted by all that heat could-a part of it-be made to do work by parting with some of it to a cooler body. The question is whether this last part, which has been imparted to a cooler body, can be restored or transferred to the warmer body again without the expenditure of energy. Rankine evidently believed such a transfer possible; for, in a paper on the "Reconcentration of the Mechanical Energy of the Universe," he has supposed it possible to reflect radiations in such a way as to give the universe such differences of temperature as to insure it a new lease of life. Clausius, in his admirable paper on the "Concentration of Rays of Light and heat," has shown

${ }^{1}$ Philosophical Magazine (4) 4, 358 .

${ }^{2}$ Mechanical Theory of Heat, Chapter XII. 
the general impossibility of such a reconcentration as Rankine supposed, when the radiating bodies are at rest; nevertheless, no such impossibility may finally appear in case of the actual universe, which is a system of moving bodies.

The law of the dissipation of energy has been applied to the universe at large, and if the consequences which have been drawn from its supposed validity are to be regarded as no longer expressing a necessary law, then we are led to affirm that without a change in the laws of nature, as at present known to us, it is possible for increasing differences of temperature to be caused without the expenditure of energy, however improbable the supposition may be that such is the fact, and however improbable it may be that such differences are actually being caused on a scale sufficient to interfere in any practical way with the progress of the dissipation of energy, as affirmed by Thomson, or check the increase of the entropy of the universe, as stated by Clausius. Still it may be remarked that a large part of the exchange of heat in the universe takes place in the radiant form; and, it seems to me, that it remains to be proved what the fact actually is, and consequently I must regard it as still an open question, as to whether on the whole the available energy of the universe is being dissipated, and its entropy increases or not.

Lest the foregoing remarks should be construed as in any sense undervaluing the splendid discoveries of Clausius, Thomson, and Rankine in the domain of thermo-dynamies, let me disclaim such an interpretation entirely, and say that my only wish is to add, if possible, to the exactness and completeness of those theories, which are among the most important of modern physies.-University of Cincinnatr, April \&2, 188\%.

NotE.-Professor J. Willard Gibbs has suggested to me that we are not at liberty to assume that reflections or radiations taking place at moving surfaces follow the same laws as from surfaces at rest; and that a perfect reflector, moving in a medium through which luminous waves are being propagated, may suffer a resistance which would require the expenditure of as much energy as could be obtained by the proposed process. Admitting for the moment the justness of these observations respecting reflections and radiations from moving surfaces, $I$ shall hope to show, in the first place, that the syren may be so adjusted that no such resistance need be encountered, and in the second place, that it is possi- 
ble so to modify the syren that no reflections or radiations need take place from moving surfaces.

In the discussion of the first point, let us consider the case of a ray falling perpendicularly upon a perfect reflector. The only numerical magnitudes susceptible of variation in this radiation are its wave length and amplitude, the velocity being assumed constant and dependent upon the elasticity of the medium. When the reflector moves in its own plane at right angles to the ray, it cannot, apparently, be seriously urged that the reflected ray will have either its wave length or its amplitude changed by the reflection. For, so far as can be seen, the wave length would suffer a change and be shortened only by giving the reflector a motion toward the approaching ray, thus crowding the waves together. Neither would the amplitude be changed, for to do this would require the moving plane to impart tangential impulses to the ether, such as can be compounded with the transverse motions already existing. If such be the tangential action of the moving plane on the ether, we should be led to the apparently inadmissible result, that since a moving plane may impart tangential impulses to the luminferous ether, a disk rotating with sufficient velocity in vacuo would become self-luminous.

It would seem but reasonable, in our present imperfect knowledge of the subject, to conclude that the only resistance which a perfect reflector experiences while moving against a ray is normal to its surface, and to be represented by a normal pressure. Even if this view be not regarded as entirely correct, it may, nevertheless, be confidently affirmed that the tangential must be small compared with the normal resistance, just as a frictional resistance of a gas is small compared with that arising from direct pressure upon a body moving through it. Hence it is seen that in spite of friction, it is possible to make a ray turn a mill whose vanes are perfect reflectors, in the same manner as the wind furns a wind-mill; and the energy expended will in that case be withdrawn from the ray itself. Now the rotating screen $c$ of the syren may be regarded as such a mill, the surfaces of whose vanes may be so inclined as to return radiations coming from $B$, partly to apertures in front of those from which they emanated, and partly to those behind, so as to exert no force either to accelerate or retard $c$. Should, however, energy be expended in moving $c$ against the reflected ray, this energy must exist immediately after the reflection in the reflected ray, and be transmitted by it to $B$. Hence we are led to the following remarkable result: On the hypothesis that radiations cause 
pressure at surfaces at which they suffer total reflection, a part of the energy of the radiation may be expended in moving the reflector against a resistence, while the remainder is all reflected to the body from which it emanated. It is to be noticed that this process of the reflecting mill, or mill as it may be called for brevity, is, if possible, in more pronounced and unequivocal contradiction to the second law than that of the syren. For the latter calls in question the accepted law of mutual exchanges, and the second law as depending upon it; but the former applies to a single body alone, as $B$, and a moving reflector. For example, let $B$ have no radiations except those through the apertures $b$; then if that part of its radiations which are not expended in turning $c$ are returned to it, it, is possible for the mill $e$ to be turned by radiations from $B$, until the energy of $B$ is all expended in performing work, thus withdrawing all heat from $B$, while no heat has been transferred to any other body in the manner required by the second law, and this regardless of the temperature of surrounding objects. It therefore seems to me that the supposition of a pressure at reflecting surfaces is more dirsetly opposed to the second law than that of no pressures.

In regard to the second point mentioned, it seems quite possible to construct a syren such that the reflections in it shall all take place from stationary surfaces, or from those whose velocity differs from zero by less than any assignable quantity. For, let the mean velocity $u$ of the screens be the same as before, but not continuons. Instead, let its motion consist of sudden steps forward, each of which is half the width of an aperture. The possibility of a mechanical arrangement, which could effect this motion without expenditure of energy, with the aid of perfect springs, fly-wheels, detents, etc., to any required degree of approximation, will, I think, be admitted, certainly by any one who can admit that Maxwell's " sorting demon " expends no energy in opening and closing apertures. It will be seen that the reflections all take place from screens at rest (or nearly so) in this modified syren, and that the same transmissions occur through its apertures as have heretofore been supposed to take place. I am not inclined, however, to insist on the special kind of apparatus which I have proposed for rendering sensible the phenomenon which I believe to exist during the time in which radiations are in process of becoming established, as contemplated in the ordinary law of thermal exchanges. The point to which $I$ would emphatically direct attention is, that since radiations 
are known to be moving in space apart from ponderable bodies, and subject to reflections, it is possible so to deal with them as to completely alter their destination, and successfully interfere with all results flowing from Prevost's law of Exchanges. It also seems to me that the exactness of the second law of thermo-dynamics depends, as far as radiations are concerned, upon that of this law of exchanges.

Cincinnati, May 18, 1882.

H. T. E.

\title{
CRANK PINS OF MARINE ENGINES.
}

\author{
Ву J. Н. Wнинам, \\ Cadet Engineer, U. S. N.
}

In compiling appended table, the co-efficient of friction was assumed to be 0.05 . The table contains crank-pin data from the modern English and American naval and merchant vessels. In each case the engines were performing well and at full power. The Miantonomah is the only exception, and she has not as yet had a fair trial of 'her machinery, but her crank-pin should run cool, as she has an excessive margin of safety, both as regards the projected area of pin and its pressure and the work of friction per unit of area. Comparing the performances of English and American crank-pins, it is seen that the work of friction and pressure per square inch of projected area for the former exceed those of the latter; also, that no one method has been followed in designing each of them.

On page 70, Prof. Marks' treatise on "The Steam Engine," the work of friction per square inch of projected aréa is given as 49,908 inch-pounds, while in the cases illustrated in table appended, the work is $12 \times 3,309.9=39,718.8$ inch-pounds, or a margin of area over 10 per cent. If, however, the averages of the best ten performances shown in the table are taken, we have work of friction per square inch projected area equal to $12 \times 5,086 \cdot 6=61,039 \cdot 2$, or the margin is exceeded by over 10 per cent.

The practice recommended in designing crank-pins is to deduce the length as shown in $\$ 30$ of Prof. Marks' treatise on "Steam Engine," and then the diameter as illustrated in $\$ \S 32,33$. A check to the result thus obtained is illustrated in the following formula, in which $c=$ coefficient of friction of rubbing surfaces; $v=$ velocity of rub- 